\title{
Breakthrough capability for UVOIR space astronomy: Reaching the darkest sky
}

\author{
Matthew A. Greenhouse ${ }^{\mathrm{a}, *}$, Scott W. Benson ${ }^{\mathrm{b}}$, Jacob Englander ${ }^{\mathrm{a}}$, Robert D. Falck ${ }^{\mathrm{b}}$, \\ Dale J. Fixsen ${ }^{\mathrm{c}}$, Jonathan P. Gardner ${ }^{\mathrm{a}}$, Jeffrey W. Kruk ${ }^{\mathrm{a}}$, Steven R. Oleson ${ }^{\mathrm{b}}$, \\ Harley A. Thronson ${ }^{\mathrm{a}}$ \\ ${ }^{\text {a } N A S A ~ G o d d a r d ~ S p a c e ~ F l i g h t ~ C e n t e r, ~ G r e e n b e l t, ~ M D ~ 20771, ~ U S A ~}$ \\ ${ }^{\mathrm{b}}$ NASA Glenn Research Center, Cleveland, OH 44135, USA \\ ${ }^{\mathrm{c}}$ University of Maryland, College Park, MD 20742, USA
}

Received 5 June 2014; received in revised form 8 October 2014; accepted 12 November 2014

Available online 21 November 2014

\begin{abstract}
We describe how availability of new solar electric propulsion (SEP) technology can substantially increase the science capability of space astronomy missions working within the near-UV to far-infrared (UVOIR) spectrum by making dark sky orbits accessible for the first time. We present a proof of concept case study in which SEP is used to enable a $700 \mathrm{~kg}$ Explorer-class observatory payload to reach an orbit beyond where the zodiacal dust limits observatory sensitivity. The resulting scientific performance advantage relative to a Sun-Earth L2 point orbit is presented and discussed. We find that making SEP available to astrophysics Explorers can enable this small payload program to rival the science performance of much larger long development-time systems. We also present flight dynamics analysis which illustrates that this concept can be extended beyond Explorers to substantially improve the sensitivity performance of heavier $(7000 \mathrm{~kg}$ ) flagship-class astrophysics payloads such as the UVOIR successor to the James Webb Space Telescope by using high power SEP that is being developed for the Asteroid Redirect Robotics Mission.

Published by Elsevier Ltd. on behalf of COSPAR. This is an open access article under the CC BY license (http://creativecommons.org/ licenses/by/3.0/).
\end{abstract}

Keywords: Solar electric propulsion; Zodiacal light; Space astronomy

\section{Introduction}

The Earth is imbedded in a cloud of dust grains that are produced by comet outgassing and impact fragmentation of asteroids that surround the inner planets. This cloud occupies a disk-shaped region of interplanetary space encompassing much of the inner solar system. It extends from approximately the orbit of Venus to the asteroid belt, and its thickness extends roughly $0.5 \mathrm{AU}$ above and below

* Corresponding author at: Laboratory for Observational Cosmology, Mail Code 443.2, Goddard Space Flight Center, Greenbelt, MD 20771, USA. Tel.: +1 3012864517 .

E-mail address: matt.greenhouse@nasa.gov (M.A. Greenhouse). the ecliptic plane (Fig. 1). This interplanetary dust (IPD) cloud produces a UVOIR background light, known as the zodiacal light, through which all space observatories have had to observe. This background light (which is often brighter than the objects being observed) is a source of noise that typically limits the sensitivity of all space astronomy imaging systems that have operated within the nearUV to far-infrared spectrum.

As development of UVOIR photon counting detectors advance in technology readiness for spaceflight application, sources of electrical noise become vanishingly small (cf: Mazin et al., 2013; Nikzad et al., 2012; Wen et al., 2006; Perryman et al., 1999; Romani et al., 1999). As a consequence, in the coming decade, photon noise from the 


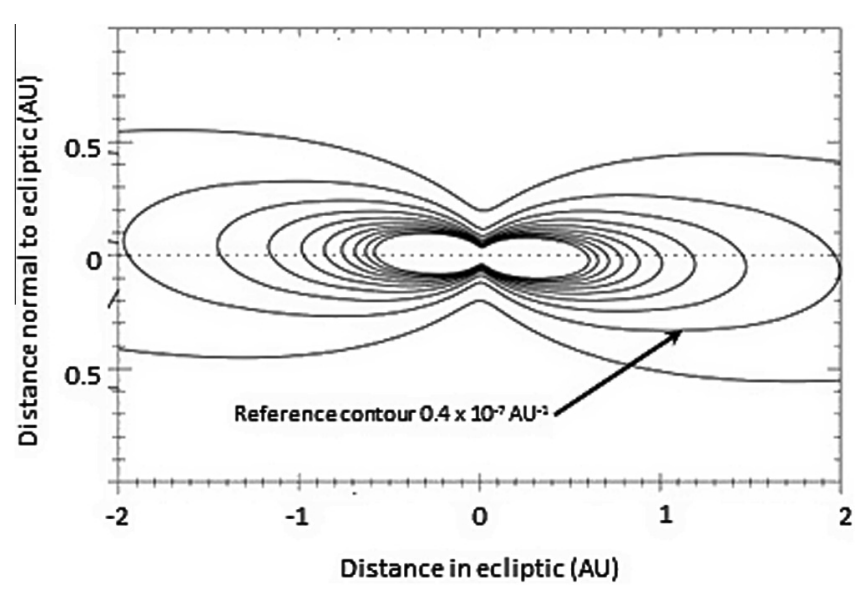

Fig. 1. Top: Isodensity contours of the interplanetary dust cloud on a plane perpendicular to the ecliptic plane from Kelsall et al. (1998); The outer most contour, in units of inverse mean free path, is $0.2 \times 10^{-7} \mathrm{AU}^{-1}$ and the contour interval is 0.2 . In this paper we take IPD density $<0.4 \times 10^{-7} \mathrm{AU}^{-1}$ (denoted by the indicated reference contour) as being "extra zodiacal". No UVOIR astrophysics mission has heretofore achieved an extra-zodiacal orbit.

IPD will become the primary limit on space observatory performance across the UVOIR spectrum for both broad-band and spectroscopic applications. In this paper, we discuss how this limit can be overcome through application of advanced in-space propulsion technology to astrophysics missions.

Sunlight that impinges on the IPD is both scattered and absorbed. Near the Earth, at wavelengths shortward of $\sim 3 \mu \mathrm{m}$, the zodiacal background light is dominated by the scattered component, and its spectral characteristics are that of the Sun. In the plane of the ecliptic, the space density of the grains decreases with distance $r$ from the Earth roughly as $1 / r$. So the scattered component decreases with distance as a result of both the decreasing grain space density and $1 / r^{2}$ dilution of the sunlight itself. The sunlight that is absorbed by the grains heats them to a temperature of roughly $240 \mathrm{~K}$ at $1 \mathrm{AU}$ (Fixsen and Dwek, 2002) resulting in blackbody emission peaking at a wavelength of approximately $12 \mu \mathrm{m}$. Near the Earth, this blackbody component dominates the zodiacal background at wavelengths longward of $\sim 3 \mu \mathrm{m}$. A minimum in the brightness of the total background emission occurs near $3 \mu \mathrm{m}$ where scattering and self-emission exchange roles as the dominant emission mechanism (Fig. 2). However, the grain temperature decreases with distance from the Sun causing the wavelength of this minimum to increase with distance beyond 1 AU reaching approximately $4.5 \mu \mathrm{m}$ at $2 \mathrm{AU}$.

Architecting a space astrophysics mission to operate in a low density region of the IPD and hence, reach the darkest sky, can follow two general approaches. One can design an orbit in the ecliptic plane with apogee in the outer solar system, or one can take advantage of the disk morphology of the IPD (Fig. 1) and utilize a lower apogee orbit that is inclined with respect to the ecliptic plane. There is a continuous trade space between them for optimization of a given astrophysics mission objective.

High apogee in-plane orbits can reach an environment characterized by grain density and temperature that is low relative to a $1 \mathrm{AU}$ orbit such as the Sun-Earth L2 point (SEL2); thus, yielding a very large gain in observatory sensitivity performance across the UVOIR spectrum. However, from a systems perspective, this performance advantage comes with challenges in electrical power generation and telecommunications bandwidth. Lower-apogee, high-inclination orbits ease these challenges but at the expense of propellant needed to achieve them.

In the following sections we discuss a case study involving use of electric propulsion to enable an Explorer-class astrophysics observatory to reach the darkest sky. In Section 2, we describe highlights of a feasibility study for a

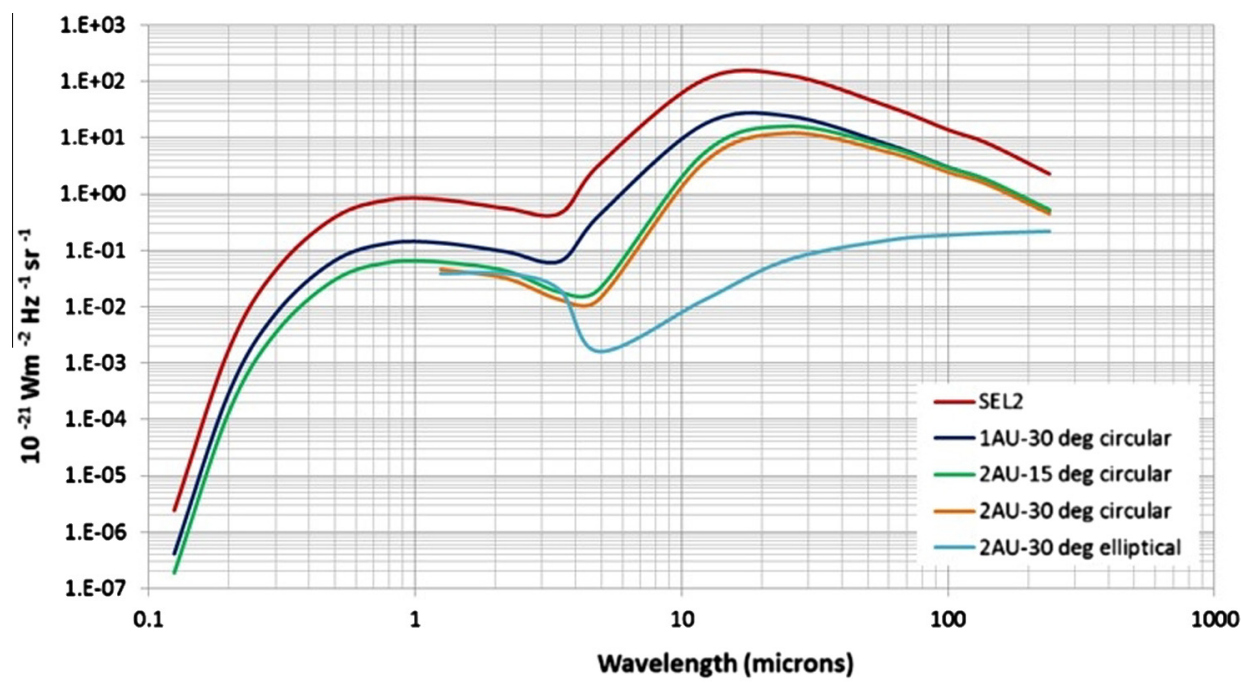

Fig. 2. The zodiacal background flux density toward the ecliptic pole for five heliocentric orbit cases. The curves shown are a spline fit to ten filter bands of the COBE Diffuse Infrared Background Experiment (DIRBE) spanning the $1-240 \mu \mathrm{m}$ spectrum, and are evaluated at the point most distant from the ecliptic plane in each inclined orbit. A $5600 \mathrm{~K}$ blackbody was used to extrapolate these data to the optical and near-UV spectrum. 


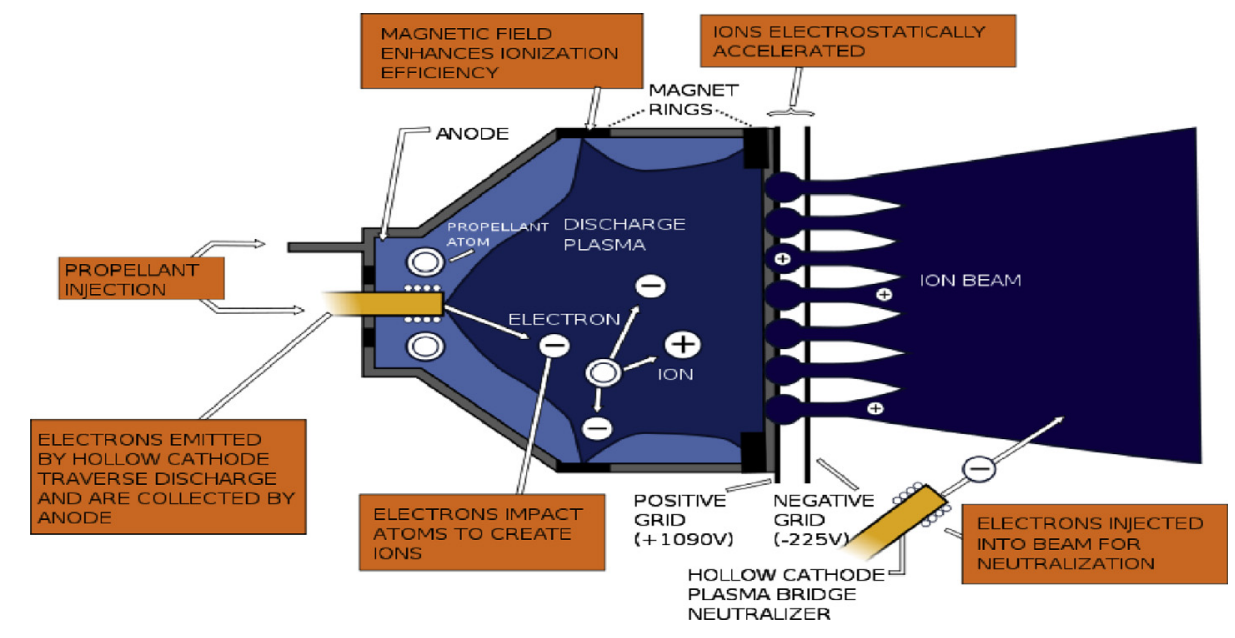

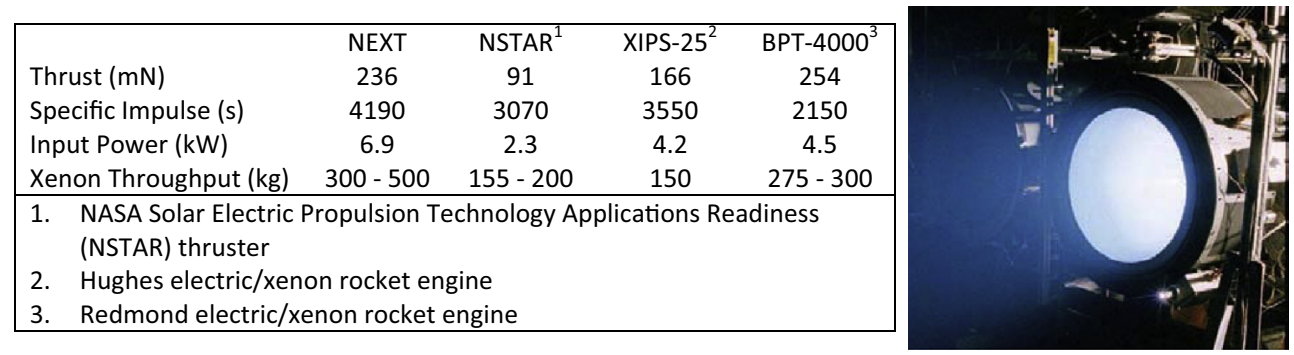

Fig. 3. NASA's Evolutionary Xenon Thruster (NEXT) uses electrical energy produced by solar panels to accelerate Xenon ions producing thrust in a way that is much more efficient than conventional chemical thrusters (top). A NEXT is shown operating in a hot fire test (bottom right). NEXT has demonstrated lifetime in ground testing that is $2 \mathrm{X}$ that required for the mission applications discussed here. Performance properties achieved by NEXT are shown along with those of other similar power SEP thrusters (bottom left). The propulsion module discussed in this paper utilizes a two NEXT thruster system that can throttle across an input power range of $12.5 \mathrm{~kW}$ (at $1 \mathrm{AU}$, with 2 thrusters) to a minimum of $725 \mathrm{~W}$ with a single thruster.

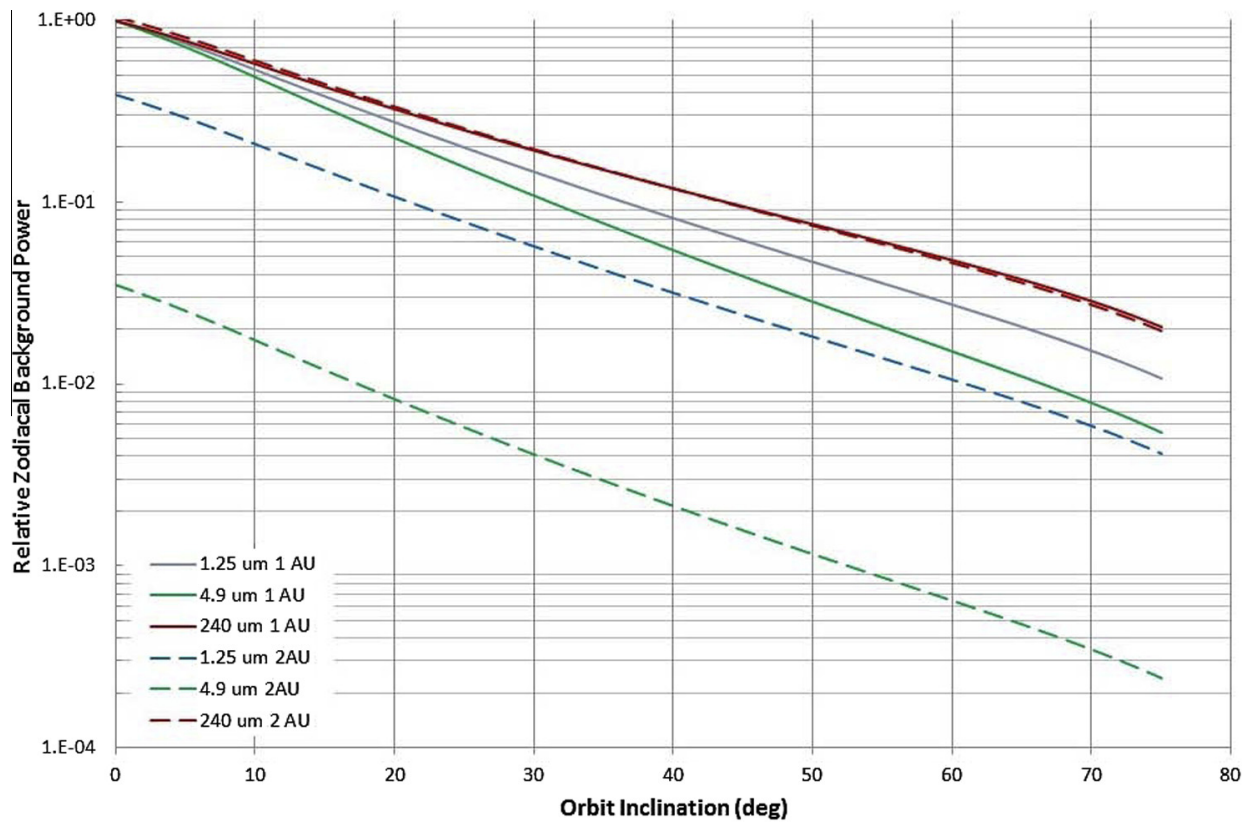

Fig. 4. Zodiacal background power toward the ecliptic pole relative to the SEL2 point vs inclination with respect to the ecliptic plane. Three DIRBE bands are shown for heliocentric radii of 1 (solid) and $2 \mathrm{AU}$ (dashed). The background power is evaluated at the maximum distance from the ecliptic plane. 


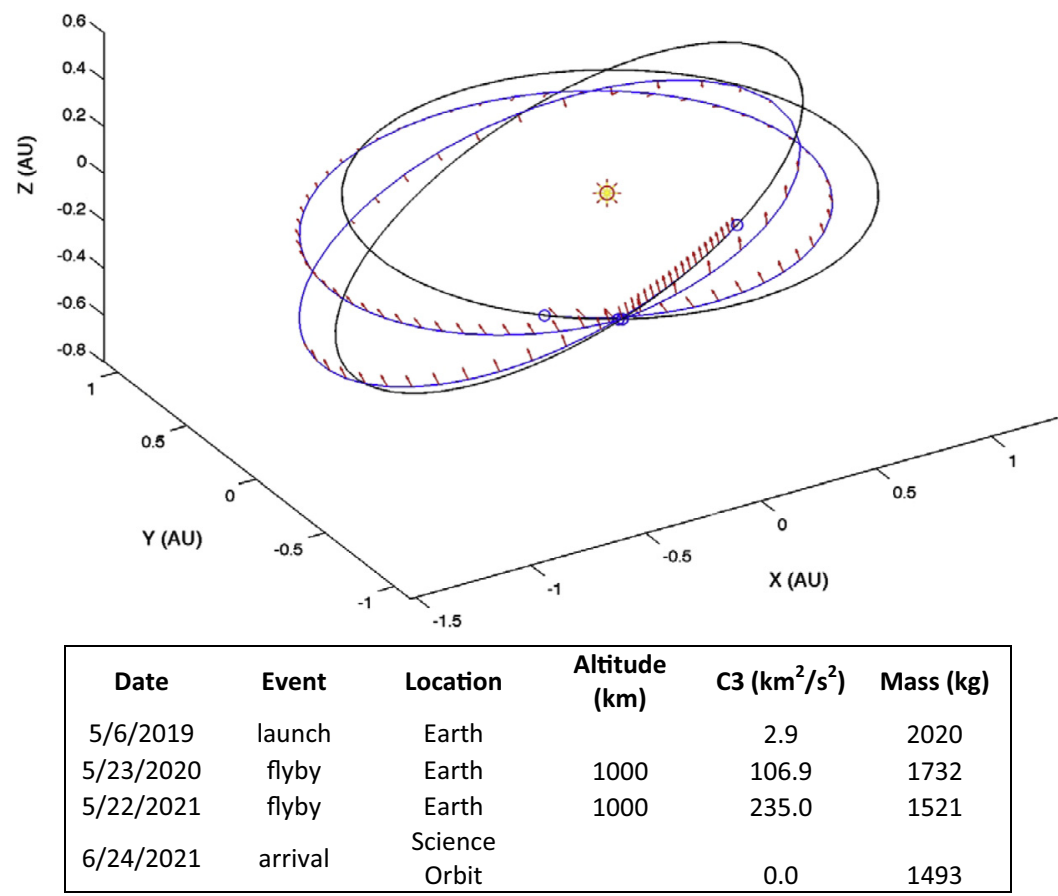

Fig. 5. Transfer orbit for $1 \mathrm{AU} 30 \mathrm{deg}$ circular science orbit case. See Benson et al. (2011) for orbit elements. The blue curve denotes the spacecraft trajectory. Red arrows indicate the direction of SEP thrust. Orbits of the Earth, and the final science orbit are shown in black. The NEXT system is throttled with available power throughout the transfer orbit. (For interpretation of the references to colour in this figure legend, the reader is referred to the web version of this article.)

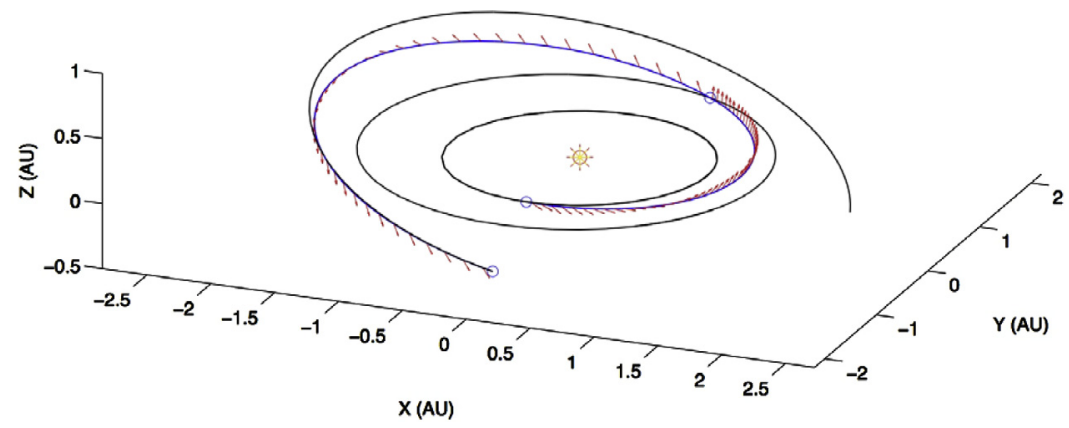

\begin{tabular}{|cccccc|}
\hline Date & Event & Location & Altitude $(\mathbf{k m})$ & $\mathbf{C 3}\left(\mathbf{k m}^{\mathbf{2}} / \mathbf{s}^{\mathbf{2}}\right)$ & Mass $(\mathbf{k g})$ \\
$6 / 20 / 2020$ & launch & Earth & & 6.2 & 1823 \\
$1 / 16 / 2021$ & flyby & Mars & 1000 & 12.3 & 1653 \\
$6 / 22 / 2022$ & arrival & Science Orbit & & 0.0 & 1439 \\
\hline
\end{tabular}

Fig. 6. Transfer orbits for the 2 AU 15 deg circular science orbit case. See Benson et al. (2011) for orbit elements. The blue curve is the spacecraft trajectory. Red arrows indicate the direction of SEP thrust. Orbits of the Earth, Mars and the final science orbit are shown in black. The NEXT system is throttled with available power throughout the transfer orbit. (For interpretation of the references to colour in this figure legend, the reader is referred to the web version of this article.)

notional Explorer-class dark sky astrophysics mission architecture (aspects of which have appeared in non-refereed conference proceedings referenced below). In Section 3, we discuss programmatic means by which this propulsion technology can be applied to enable community proposed Explorer- and Discovery-class missions to reach deep space. Conclusions are summarized in Section 4. In Appendix A, we look beyond Explorer-class mission application and present flight dynamics analysis which illustrates the potential of high power electric propulsion systems being developed for deep space human exploration to enable a $7000 \mathrm{~kg}$ flagship-class astrophysics mission to reach dark sky.

No UVOIR astrophysics mission has heretofore achieved an extra-zodiacal orbit. To our knowledge, this the only journal article describing the potential of extrazodiacal astronomy or how such a mission could be practically realized. 


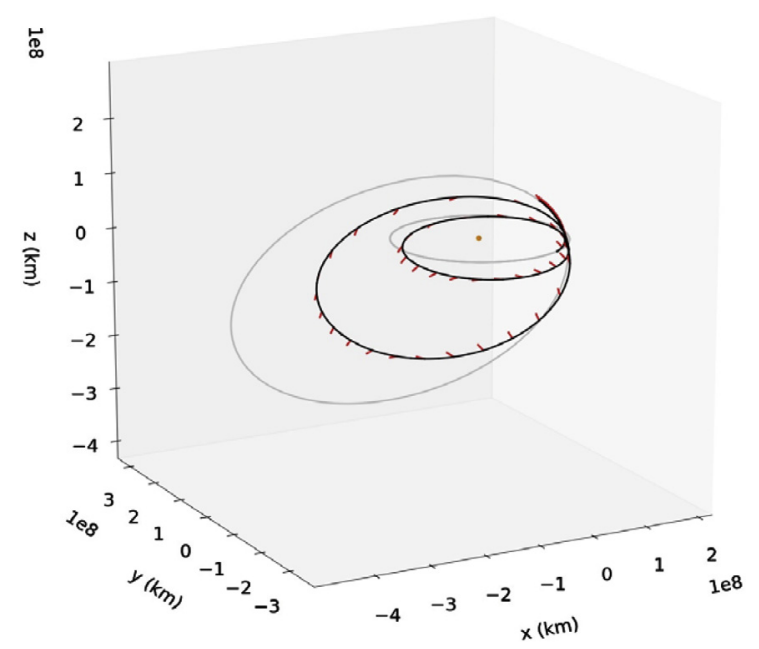

$\begin{array}{lc}\text { Semi-major axis (AU) } & 2 \\ \text { Eccentricity } & 0.56 \\ \text { Incination (deg) } & 30 \\ \text { Right asention of the assending Node } & 316 \\ \text { (deg) } & 51 \\ \text { Argument of perihelion (deg) } & 2.82 \\ \text { Period (years) } & 3.15 \\ \text { Flight Time to Arrival (years) } & \end{array}$

\begin{tabular}{|cccccc|}
\hline Date & Event & Location & Altitude $(\mathbf{k m})$ & $\mathbf{C 3}\left(\mathbf{k m}^{\mathbf{2}} \mathbf{\mathbf { s } ^ { 2 }}\right)$ & Mass $\mathbf{( k g )}$ \\
$7 / 21 / 2020$ & launch & Earth & & 12.5 & 2116 \\
$8 / 9 / 2021$ & flyby & Earth & 300 & 137.3 & 1801 \\
$8 / 8 / 2023$ & flyby & Earth & 300 & 379.6 & 1474 \\
$9 / 15 / 2023$ & arrival & Science orbit & & 0 & 1439 \\
\hline
\end{tabular}

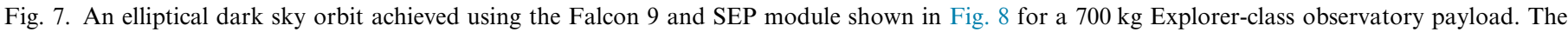

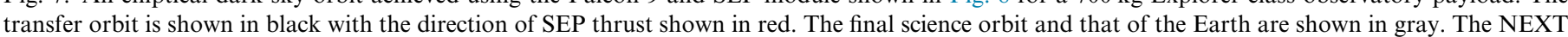
system is throttled with available power throughout the transfer orbit.

\section{The extra-zodiacal Explorer (EZE)}

The EZE refers to a detailed mission architecture study (Benson et al., 2011; Greenhouse et al., 2012) that was conducted jointly by the Goddard Space Flight Center and the Glenn Research Center (GRC) using the GRC COllaborative Modeling for Parametric Assessment of Space Systems (COMPASS) facility to determine if and how a technically mature SEP system, such as the NASA Evolutionary Xeon Thruster (NEXT) shown in Fig. 3, can be used to enable astrophysics Explorer missions to operate in an IPD density environment that is very low relative to the SEL2 point in order to increase the scientific potency of this small payload program.

Two heliocentric inclined circular orbit cases of radius 1 and 2 AU were considered in detail. The dependence of the zodiacal background on orbit inclination with respect to the ecliptic plane is shown in Fig. 4. Inclination angles of 30 and $15 \mathrm{deg}$ were chosen respectively to reach an IPD column density of $<0.4 \times 10^{-7} \mathrm{AU}^{-1}$ (Fig. 1). Flight dynamics analysis was conducted using MALTO (Benson et al., 2011). In the 1 AU case, approximately $50 \%$ of the plane-change energy was achieved via a single gravity assist encounter with the Earth. Phasing with the Earth was achieved to yield a maximum telecommunications range of 0.7 AU over the orbit period. In the 2 AU case, a single gravitational assist from Mars was utilized. The transfer orbits for these two orbit cases are shown in Figs. 5 and 6.

A 2 AU 30 deg elliptical orbit case was also considered as a means to increase the fraction of the orbital period that is spent outside of the $0.4 \times 10^{-7} \mathrm{AU}^{-1}$ contour of Fig. 1. In this case, the Evolutionary Mission Trajectory Generator (EMTG) described by Englander et al. (2013) was used to find the transfer orbit shown in Fig. 7. The zodiacal background as a function of mission time in this orbit is shown in Fig. 8 along with that of a circular orbit case with the same inclination.

As illustrated in Fig. 8, choice of an elliptical orbit can yield long contiguous periods of dark sky performance. However, as a matter of mission design, one must choose between favoring the north or south ecliptic hemisphere. In contrast, the circular cases are symmetric in this regard but afford shorter dark sky periods. The SEP cruise time and propellant mass needed to reach these example orbits are summarized in Table 1.

In all cases, we found that acceptable science data downlink rates in the range of approximately $0.5-3.5 \mathrm{Mbps}$ can be enabled by conventional Ka-band ( $32 \mathrm{GHz}$ ) flight-ready 


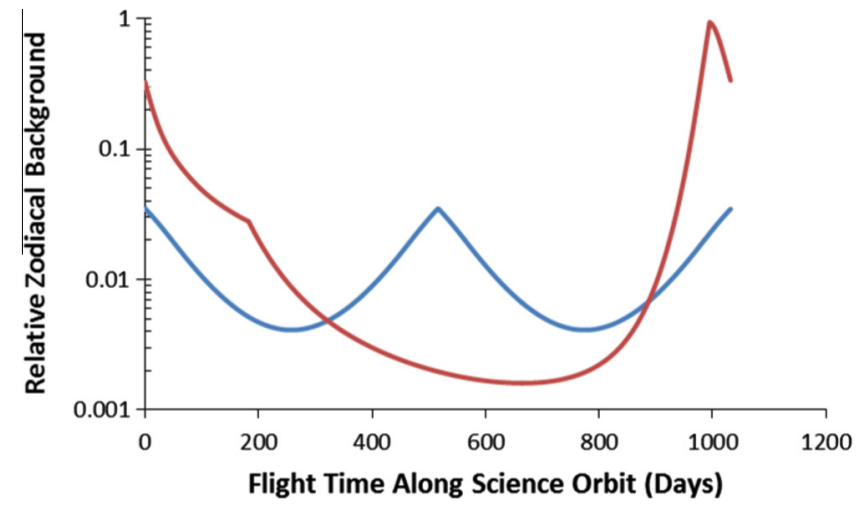

Fig. 8. The relative zodiacal background power toward the ecliptic pole as a function of orbital period is shown for the orbit of Fig. 7 (red) and a zero eccentricity orbit (circular) orbit of the same inclination and semi major axis (blue). The relative power shown is for the $4.9 \mu \mathrm{m}$ DIRBE band calculated toward the more favorable pole (north or south) depending on position with respect to the ecliptic plane. (For interpretation of the references to color in this figure legend, the reader is referred to the web version of this article.)

Table 1

Transfer orbit duration and propellant utilized for three orbit cases.

\begin{tabular}{lcc}
\hline Orbit case & SEP cruise phase (years) & Propellant used (kg) \\
\hline 1 AU 30 deg circular & 2.13 & 527 \\
2 AU 15 deg circular & 2.00 & 384 \\
2 AU 30 deg elliptical & 3.15 & 677 \\
\hline
\end{tabular}

Table 2

EZE science data down-link capability.

\begin{tabular}{|c|c|c|c|c|c|}
\hline Orbit & $\begin{array}{l}\text { Max } \\
\text { range } \\
(\mathrm{AU})\end{array}$ & $\begin{array}{l}\text { Space } \\
\text { terminal } \\
(55 \% \\
\text { efficiency) }\end{array}$ & $\begin{array}{l}\text { Ka band } \\
\text { power } \\
\text { (W) }\end{array}$ & $\begin{array}{l}\text { Ground } \\
\text { terminal }\end{array}$ & $\begin{array}{l}\text { Minimum } \\
\text { data rate } \\
(\mathrm{Mbps})\end{array}$ \\
\hline $1 \mathrm{AU} 30 \mathrm{deg}$ circular & 0.7 & $1.0 \mathrm{~m} \mathrm{HGA}$ & 200 & DSN $36 \mathrm{~m}$ & 3.5 \\
\hline 2 AU 15 deg circular & 3.0 & $1.5 \mathrm{~m} \mathrm{HGA}$ & 360 & DSN $36 \mathrm{~m}$ & 0.74 \\
\hline 2 AU 15 deg circular & 3.0 & $2.0 \mathrm{~m} \mathrm{HGA}$ & 200 & DSN $36 \mathrm{~m}$ & 0.74 \\
\hline 2 AU 30 deg elliptical & 4.0 & $2.0 \mathrm{~m} \mathrm{HGA}$ & 360 & DSN $36 \mathrm{~m}$ & 0.48 \\
\hline
\end{tabular}

technologies (Table 2). The cases shown in Table 2 utilize $200 \mathrm{~W}$ traveling wave tube amplifiers that were built for the Jupiter Icy Moons Mission (JIMO) and assume $3 \mathrm{~dB}$ of link margin after $3 \mathrm{~dB}$ of rain fade and $0.2 \mathrm{~dB}$ of pointing loss. The rates shown in Table 2 are lower limits calculated at the maximum range. An Explorer-scale observatory would typically utilize a fixed (non-gimbaled) High Gain Antenna (HGA) necessitating interruption of observing for daily science data down-links. The rates shown in Table 2 would enable data volume capability that is sufficient for typical Explorers within several hours of ground contact per day.

We showed that Falcon-9, augmented by a SEP upper stage orbit transfer module (Fig. 9) utilizing a throttled two NEXT thruster system, can place a notional $700 \mathrm{~kg}$ (Class EX) Explorer payload (Fig. 8) into any of the above orbits - thus enabling observatory performance gain shown in Fig. 9 relative to the low Earth orbit of the Hubble Space Telescope, the Earth-trailing orbit of the Spitzer and Kepler Space Telescopes, or the Sun-Earth L2 point orbit of the James Webb and Herschel Space Telescopes.

We followed a mission architecture approach in which the SEP is implemented as an upper stage module that is controlled by the science payload spacecraft and ejected via a lightband separation system (Holms, 2004) when the payload is delivered to its operational orbit (Fig. 12). This approach has several advantages.

It enables a single module design to be applied to a wide range of community proposed missions spanning both astrophysics and planetary science applications. Provision of a standardized propulsion module, as government furnished equipment along with launch services, can enable the benefits of this technology to be realized with minimum non-recurring engineering cost and technical risk. In this light, we adopted a design approach involving a clean adiabatic systems interface (Fig. 13) between the propulsion module and science payload spacecraft that Explorer program proposers could design to following a user's manual. Ability to eject the SEP module after it is no longer needed can enable payloads with stringent pointing requirements to avoid the dynamic effects of large low frequency $(\sim 1 \mathrm{~Hz})$ solar array structures. Top-level mass properties for the module shown in Fig. 9 are given in Table 3. In this table, an allowance for mass growth is included that is determined by the degree of flight heritage in the subsystem design.

The EZE module concept is consistent with a wide range of solar array systems. Our study utilized Orion Ultraflex ${ }^{1}$ arrays due to their high technical maturity. Of the $45 \mathrm{~m}^{2}$ area afforded by two $6 \mathrm{~m}$ diameter Ultraflex arrays shown in Fig. 9, we found it necessary to populate only $41 \mathrm{~m}^{2}$ with $25 \%$ efficient triple junction GaAs solar cells, in order to provide (at $1 \mathrm{AU}$ beginning of life) $12.5 \mathrm{~kW}$ to the SEP power processing units at $120 \mathrm{vdc}$ and $577 \mathrm{~W}$ to housekeeping loads at $28 \mathrm{vdc}$. The SEP module thermal control system dissipates power processor waste heat while thruster waste heat is radiated to deep space. The $13 \mathrm{~kW}$ module design shown in Figs. 9 and 10, was sized to the fault tolerance requirements of a $700 \mathrm{~kg}$ medium Explorer payload, and is scalable to higher mass or higher reliability applications.

This study demonstrated that all enabling technologies for this propulsion module currently meet technology readiness for infusion into an Explorer mission solicitation during this decade. The module designed in this study represents a substantial step forward in capability over the prior SEP flight state of art demonstrated by the Dawn mission (Russell et al., 2007), for support of a wide range of Class- $\mathrm{C}^{2}$ astrophysics or heliophysics Explorer program

\footnotetext{
${ }^{1}$ http://www.atk.com/products-services/ultraflex-solar-array-systems/.

2 http://nodis3.gsfc.nasa.gov/displayDir.cfm?Internal_ID = N_PR 8705_0004_\&page_name=AppendixB.
} 

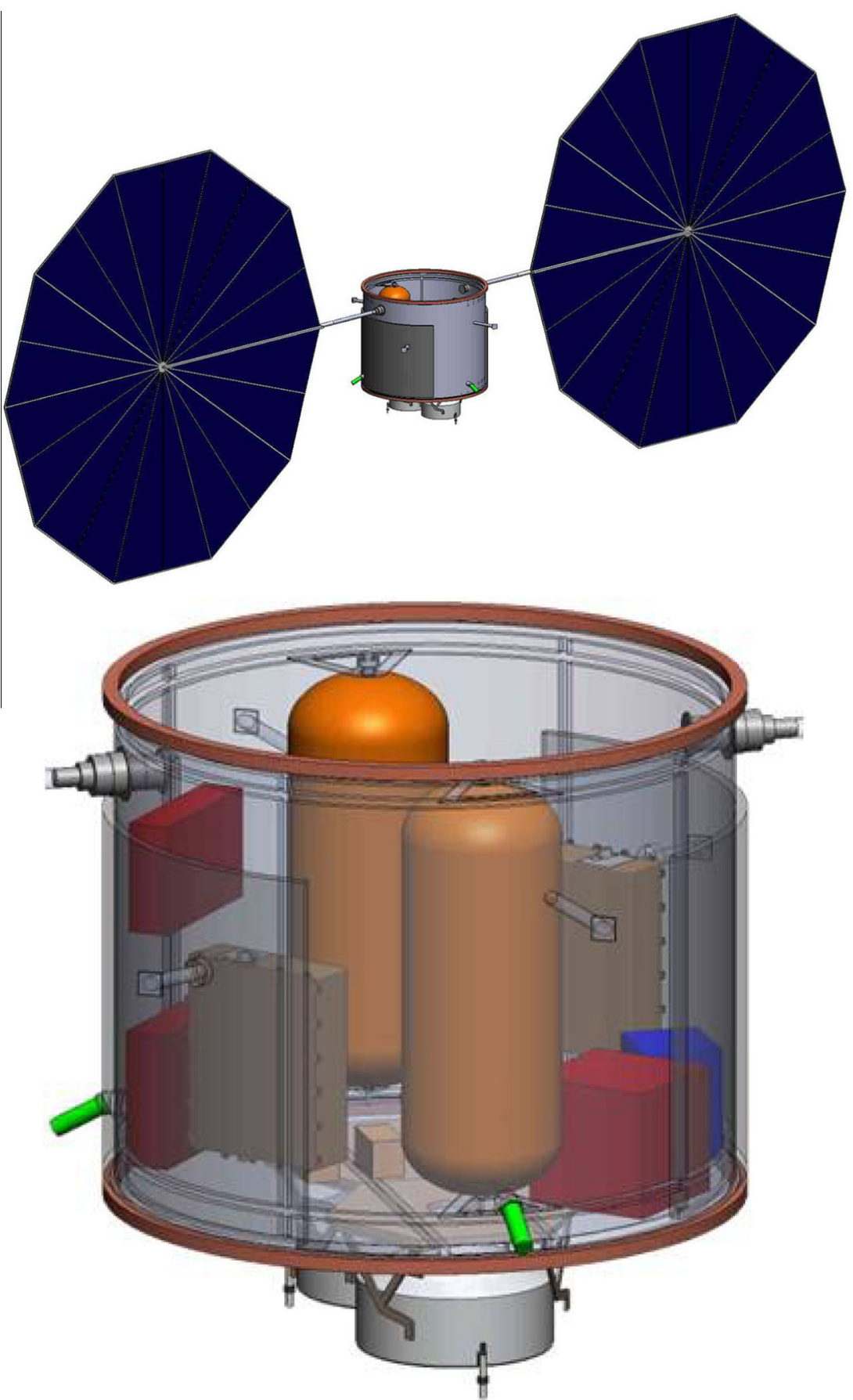

Fig. 9. The $13 \mathrm{~kW}$ EZE SEP orbit transfer module is shown in its cruise configuration with $6 \mathrm{~m}$ diameter Orion Ultraflex solar arrays deployed (top) and in detail (bottom). The cylindrical section of the module is $1.64 \mathrm{~m}$ in diameter and $1.41 \mathrm{~m}$ long. Further details of the module design are described in Benson et al. (2011).

mission applications, and is scalable to Class-B planetary science Discovery program missions.

\section{Discussion}

Cost constraints on space science necessitate maximizing the performance of community proposed Explorer-class payload programs that can provide university research teams' frequent access to space. Provision of SEP-augmented launch services such as described here, as govern- ment furnished equipment in Explorer and Discovery mission solicitations is a necessary step in ensuring the long-term scientific viability of these programs, and, as illustrated in Appendix A, will serve as a key path finding step toward enabling UVOIR flagship successors to the JWST to achieve optimal science performance per unit telescope aperture.

Full return on investment in photon counting detector development can only be realized by enabling missions that utilize them to reach the darkest sky. Making SEP technol- 

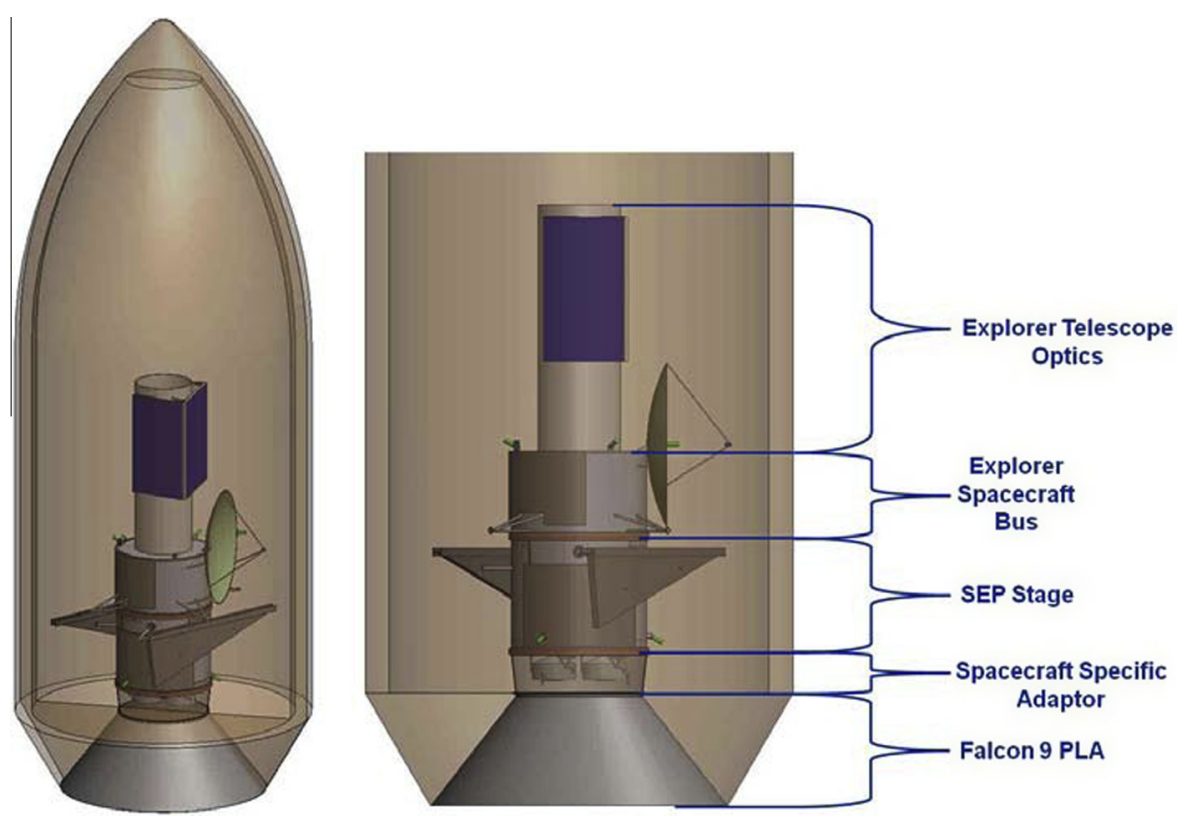

Fig. 10. Top: A notional $700 \mathrm{~kg}$ astrophysics Explorer integrated with the SEP module shown in Fig. 7 and encapsulated in a Falcon 9 faring.
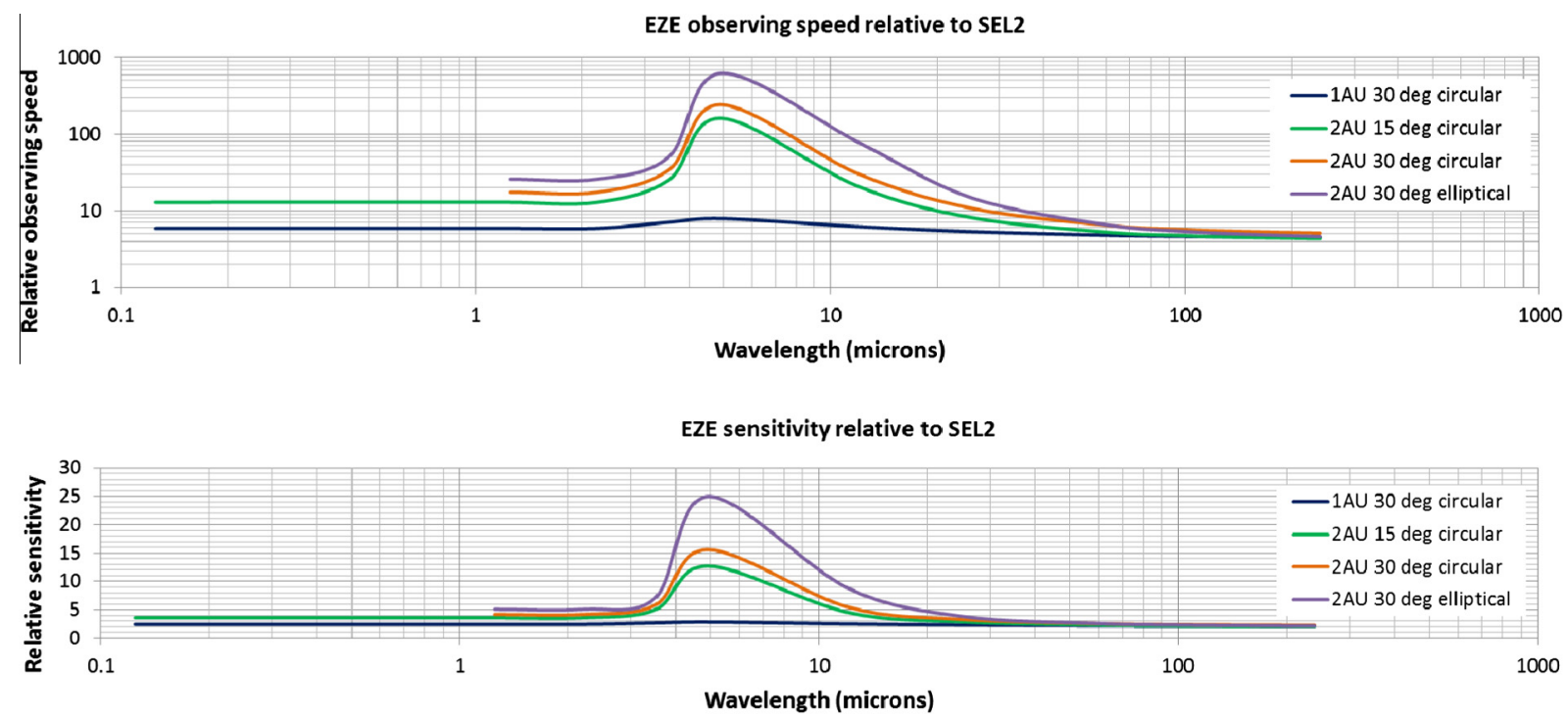

Fig. 11. The relative observing speed and point source sensitivity gain for background-limited observations in dark sky orbits enabled by the SEP module shown in Fig. 9. The relative speed and sensitivity are taken as proportional to the relative zodiacal background (Fig. 2) and square root of the relative background respectively.

ogy available to community proposed astrophysics missions in a cost effective way is the means to that end.

In the EZE study discussed above, we found that provision of SEP through development of an orbit transfer module that is infused into NASA's Explorer and Discovery programs as part of government furnished launch services is practical and cost effective - particularly from the perspective of minimizing cost to support mission applications spanning all NASA space science disciplines. We demonstrated that this approach can offer a clean systems interface to Explorer and Discovery program proposers - thus enabling build-to-print duplication of a common design for minimization of both non-recurring engineering cost and risk through successive flight heritage of a common design.

We adopted a design approach that accommodates ejection of the module upon orbit insertion in order to support missions with pointing requirements that cannot tolerate low frequency solar array structures (such as high slew rates with short settling time). However, we note that most mission applications would not require separation. Those cases would benefit from several advantages. The NEXT thrusters are gimbal-mounted, and can be used to de-spin reaction wheels in the science payload spacecraft, thus 


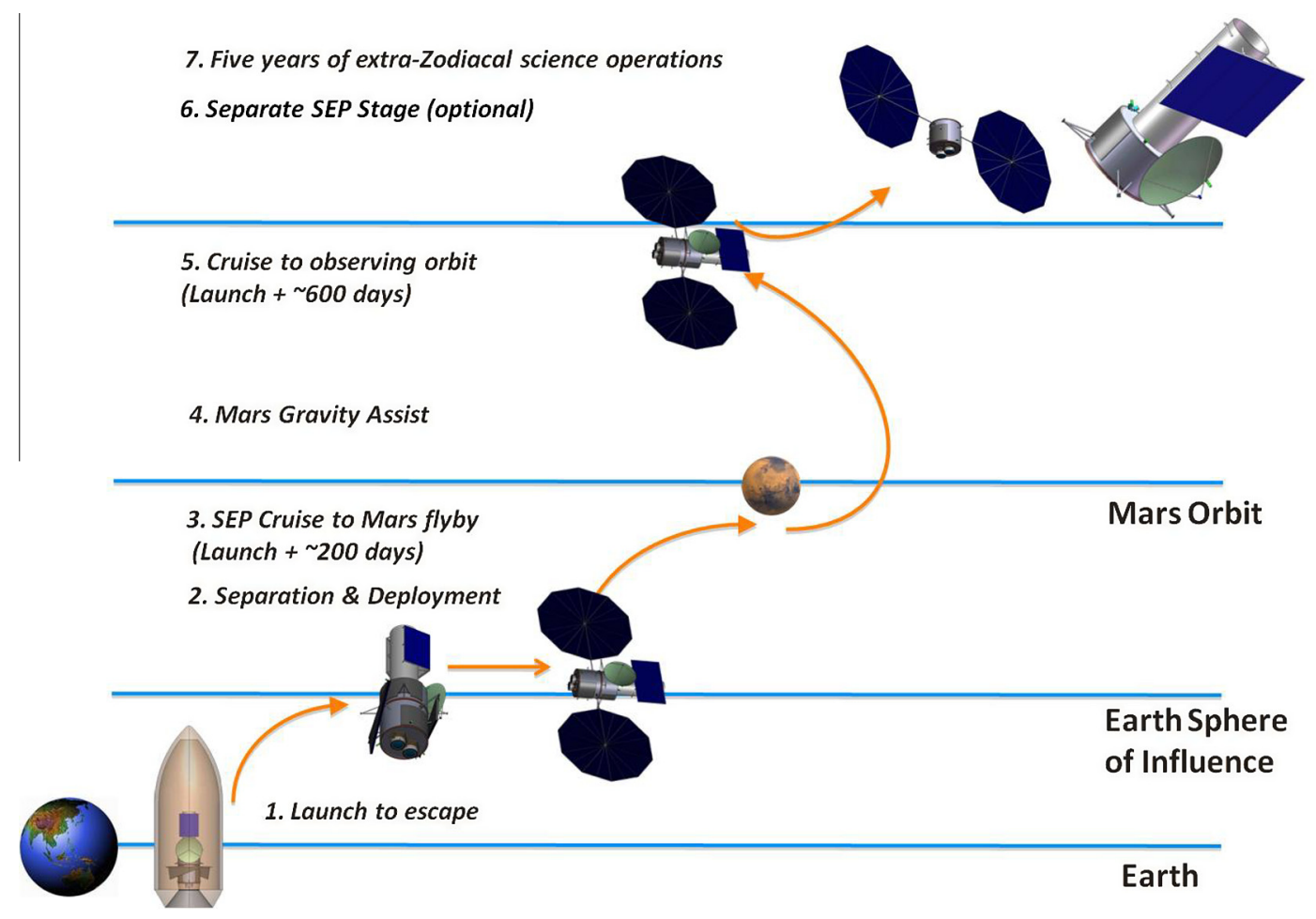

Fig. 12. The timeline sequence of events is shown for the EZE mission architecture.

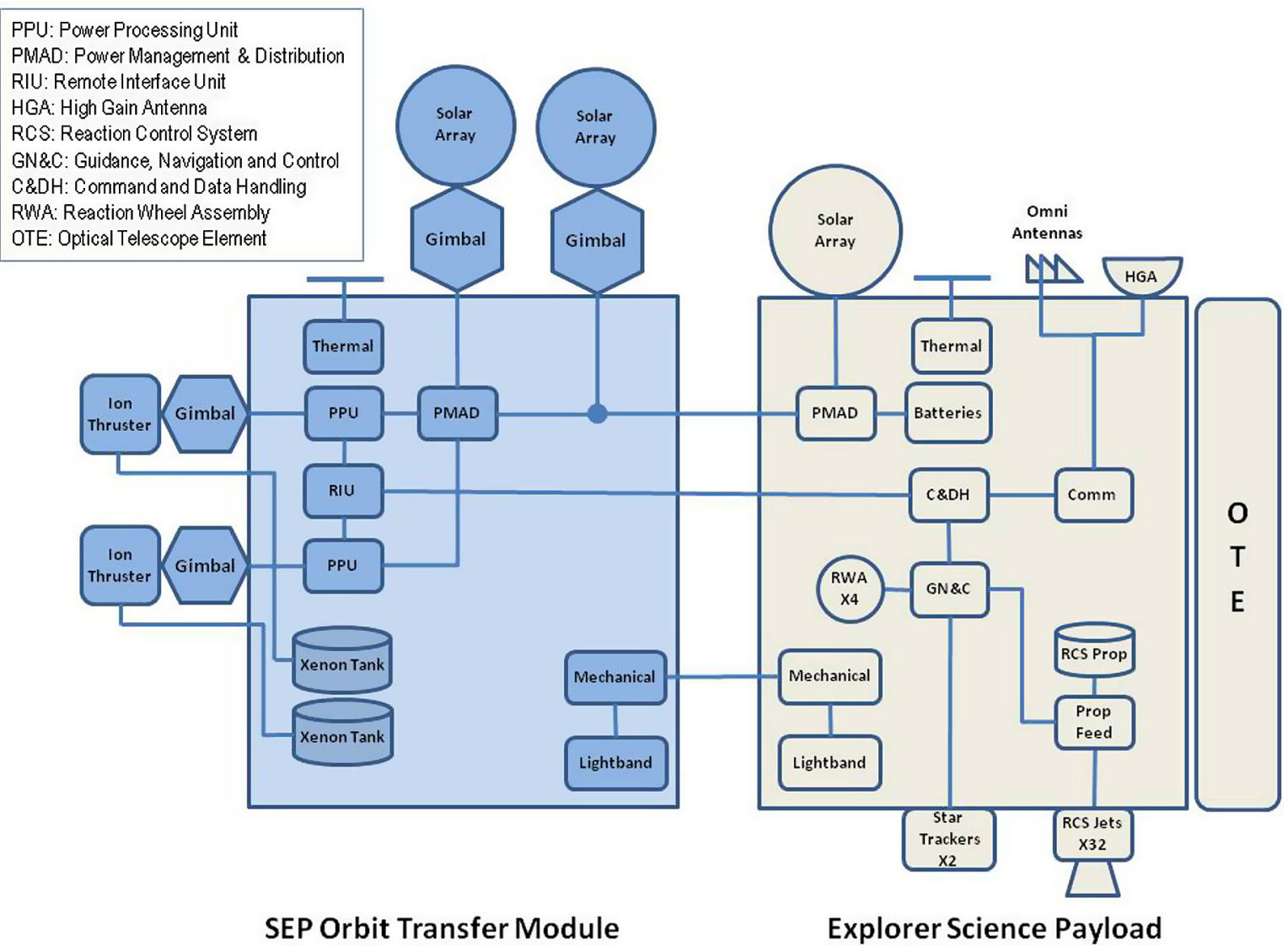

Fig. 13. The EZE SEP orbit transfer module (left) is controlled by its science payload spacecraft (right) yielding a clean systems interface to support a wide variety of community-proposed Explorer or Discovery program payloads. 
Table 3

EZE solar electric orbit transfer module mass properties.

\begin{tabular}{lccr}
\hline Main Subsystem & $\begin{array}{c}\text { Mass } \\
(\mathrm{kg})\end{array}$ & $\begin{array}{l}\text { Growth } \\
(\%)\end{array}$ & $\begin{array}{c}\text { Total } \\
(\mathrm{kg})\end{array}$ \\
\hline Attitude determination \& control & 1 & 3 & 1 \\
Remote interface unit & 35 & 29 & 45 \\
Communications \& tracking & 4 & 30 & 5 \\
Electrical power & 182 & 18 & 215 \\
Thermal control (non-propellant) & 58 & 15 & 67 \\
Propulsion \& propellant & 168 & 8 & 181 \\
$\quad$ management & 91 & 18 & 107 \\
Structures \& mechanisms & & & 622 \\
Estimated module dry mass with & & & \\
$\quad$ growth & & &
\end{tabular}

eliminating need for reaction control thrusters. After orbit insertion, excess power from the modules solar arrays can be utilized to achieve higher science data telecommunication rates than those shown in Table 2.

Long cruise time to science operations (such as shown in Table 1) is the norm in planetary science; however, it is new to astrophysics and inherent to low thrust propulsion. We note that, in a low cost program such as Explorers, the telescope and SEP thrusters cannot be pointed independently beyond the angular range of the thruster gimbals. Hence, ability to do astronomical science requiring pointing over a large field of regard would be restricted during this phase. However, looking beyond the Explorer-class application discussed here, this pointing restriction would not apply to a flagship-class dark sky mission (Appendix A) involving a gimbal-mounted or boom-deployed telescope. Design concept examples of boom-deployed configurations include ATLAST (Postman et al., 2012) and SAFIR (Lille and Dailey, 2005).

\section{Summary and conclusions}

Application of SEP to space science was designated as a priority in the NRC Decadal Survey Vision and Voyages for Planetary Science in the Decade 2013-2022 (NRC, 2011). It is a key enabling technology for human exploration of Mars (NRC, 2012). The work described in this paper illustrates the potential of this technology toward enabling very high performance UVOIR astrophysics missions. In a literal sense, it has never been "dark time" for UVOIR space astronomers. Application of SEP to astrophysics can change that. In this paper, we illustrate an orbit transfer module design approach that can enable small

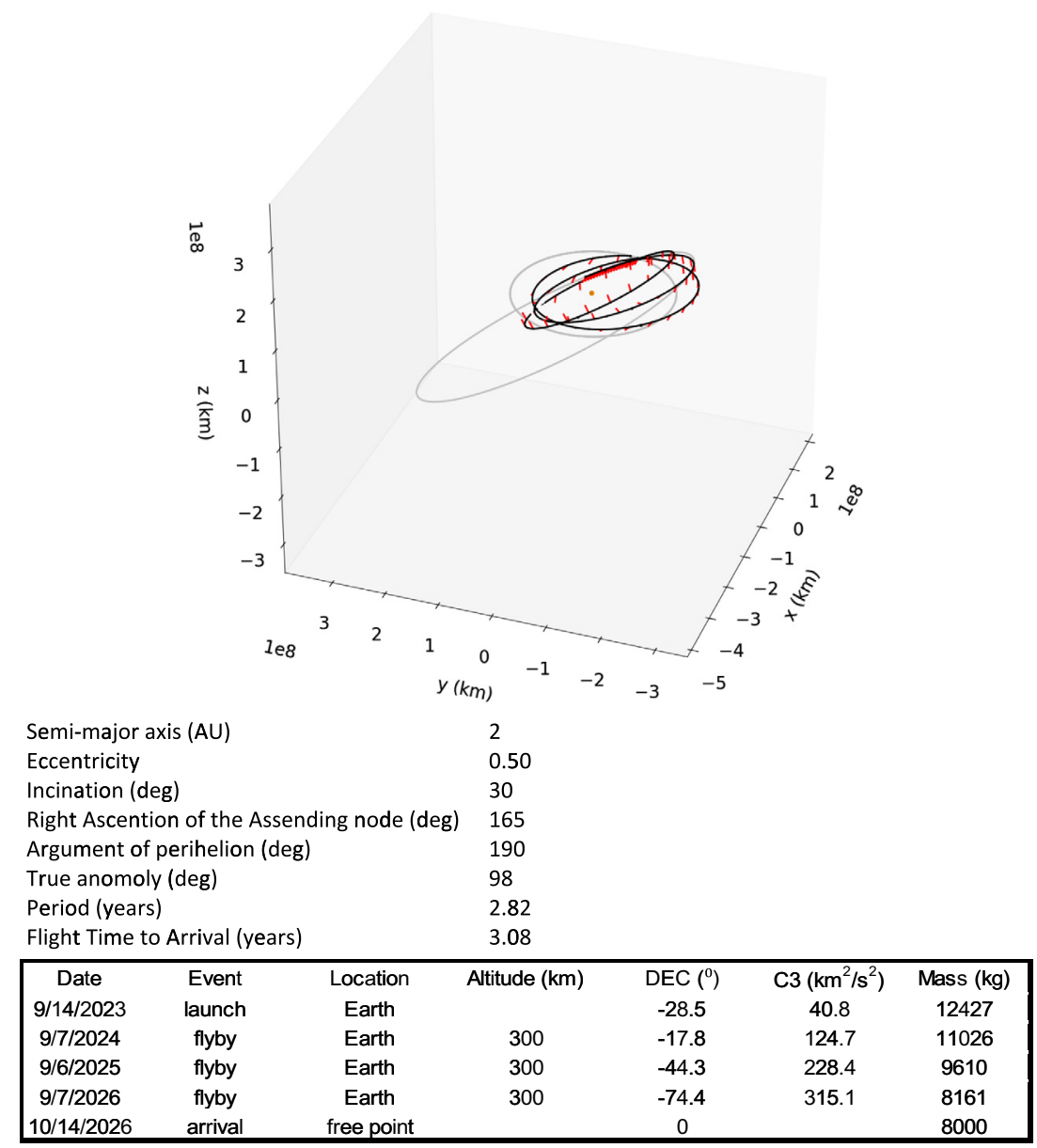

Fig. 14. An elliptical dark sky orbit achieved using the SLS Block 1a and ARRM-class SEP system (Table 4) for a flagship-class $7000 \mathrm{~kg}$ science payload. The science performance gain afforded by this orbit is shown in Fig. 11. The space vehicle arrival mass includes a $1000 \mathrm{~kg}$ dry mass allowance for the SEP system. 
Table 4

ARRM-class SEP system parameters assumed for the mission case shown in Fig. 14.

\begin{tabular}{ll}
\hline Array power & $50 \mathrm{~kW}(10 \%$ margin $)$ \\
Bus power during SEP cruise & $1 \mathrm{~kW}$ \\
Hall thruster power & $40 \mathrm{~kW}$ total $(90 \%$ duty cycle $)$ \\
ISP (s) & $3000(60 \%$ efficiency $)$ \\
Dry mass & $1000 \mathrm{~kg}$ \\
\hline
\end{tabular}

payload programs across all NASA space science disciplines to benefit from this technology. In Appendix A, we further illustrate that launch vehicle and high power SEP systems under development for NASA's Human Exploration Program can be utilized to enable a UVOIR flagship-class JWST successor mission to reach dark sky and thus achieve optimal performance per unit telescope aperture.

This study demonstrates that that SEP, used in conjunction Falcon 9 launch services, can enable a $700 \mathrm{~kg}$ Explorer science payload to reach dark sky beyond the zodiacal background light. The study reported here utilized technologies that are currently ready for infusion into an Explorer program such that no enabling propulsion or communications technology development would be needed to begin mission development of an extra-zodiacal Explorer during this decade. We find that the observatory performance advantage, in terms of point source sensitivity or survey speed, is significant across the UVOIR spectrum and is most pronounced in the mid-infrared spectrum. We illustrate a design approach involving a SEP propulsion module that affords a clean interface to a wide range of Explorer payloads to enable support of multiple Explorer missions with minimum non-recurring engineering cost.

\section{Acknowledgments}

This work was supported by internal research and development funding of the NASA Goddard Space Flight Center and the NASA Glenn Research Center. We thank the Glenn COMPASS team for extensive engineering analysis and support.

\section{Appendix A. The flagship-class UVOIR successor to the JWST}

In the above sections, we demonstrated the feasibility of enabling Explorer-class astrophysics missions to reach deep space dark sky orbits using technically mature SEP technologies. In this appendix, we briefly address the potential of higher power SEP and the Space Launch System (SLS), that are under development to support human deep space exploration, to also enable much heavier flagship-class robotic astrophysics space vehicles to reach dark sky. To address this question, we used the EMTG to assess feasibility of placing a $7000 \mathrm{~kg}$ space vehicle into a $2 \mathrm{AU} 30 \mathrm{deg}$ inclined dark sky orbit using SEP system parameters from the Asteroid Redirect Robotics Mission (ARRM) in conjunction with the Space Launch System (SLS) block 1a.

A potential NASA astrophysics roadmap application corresponding to this case would be the Large UVOIR successor to the James Webb Space Telescope (JWST). A central science objective for this mission is exoplanet imagery and spectroscopy. Direct spectroscopy of an Earth-like exoplanet target (of order 30th magnitude) necessitates an extremely low noise sensor system that is limited by photon noise from the exoplanet's continuum emission and that from the exo-IPD that may surround it. As illustrated in Fig. 11, maximizing point source sensitivity for this application or others, such as deep cosmology imagery, is facilitated by choice of a dark sky orbit in which the noise contribution of our solar system's IPD is eliminated.

One proof of concept orbit case is shown in Fig. 14, in which a $2 \mathrm{AU}$ semi-major axis $30 \mathrm{deg}$ inclined orbit is achieved with two Earth fly-bys within a SEP cruise phase of approximately 3 years. The zodiacal background power as a function of time after arrival in the operational orbit is shown in Fig. 2 yielding performance gain shown in Fig. 11. The assumed properties for the ARRM-class SEP system are shown in Table 4.

This analysis illustrates that, from a flight dynamics perspective, dark sky orbits can be achieved by flagship-class science missions described in the NASA Astrophysics Roadmap ${ }^{3}$ using SEP and launch system technologies that are technology development priorities for NASA's human exploration program.

\section{References}

Benson, S.W. et al., 2011. Extra-zodiacal cloud astronomy via solar electric propulsion. In: Proceedings of the 32nd International Electric Propulsion Conference, Wiesbaden Germany.

Englander, J. A., Ellison, D. H., Conway, B. A., 2013. "Robust global optimization of low-thrust, multiple-flyby trajectories". In: AAS/ AIAA Astrodynamics Specialist Conference, Hilton Head, SC.

Fixsen, D., Dwek, E., 2002. The Zodiacal emission spectrum as determined by COBE and its implications. ApJ 578, 1009.

Greenhouse, M.A., et al., 2012. Breakthrough capability for the NASA astrophysics explorer program: reaching the darkest sky. In: Proc SPIE, pp. 8442.

Holms, W., 2004. The Lightband as enabling technology for responsive space. In: Proceedings of the AIAA 2nd Responsive Space Conference, Los Angeles CA.

Kelsall, T. et al., 1998. The COBE diffuse infrared background experiment search for the cosmic infrared background. II. Model of the infrared background.. ApJ 508, 44-78.

Lille, C.F., Dailey, D.R., 2005. A mission architecture for future space observatories optimized for SAFIR. In: Proc SPIE, pp. 5899.

Mazin, B.A. et al., 2013. ARCONS: A 2024 pixel optical through near-IR cryogenic imaging spectrophotometer. PASP 125, 933.

Nikzad, S. et al., 2012. Delta-doped electron-multiplied CCD with absolute quantum efficiency over $50 \%$ in the near to far ultraviolet range for single photon counting applications. Appl. Opt. 51, 365.

\footnotetext{
${ }^{3}$ http://science.nasa.gov/media/medialibrary/2013/12/20/secureAstrophysics_Roadmap_2013.pdf.
} 
National Research Council, 2011. Committee on the Planetary Science Decadal Survey, Vision and Voyages for Planetary Science in the Decade 2013-2022, National Academies Press, Washington DC.

National Research Council, 2012. Steering Committee for NASA Technology Roadmaps, NASA Space Technology Roadmaps and Priorities: Restoring NASA's Technological Edge and Paving the Way for a New Era in Space, National Academies Press, Washington DC.

Perryman, M.A.C., Favata, F., Peacock, A., Rando, N., Taylor, B.G., et al., 1999. Optical ST J observations of the crab pulsar. A\&A 346, L30.
Postman, M. et al., 2012. Advanced technology large-aperture space telescope: science drivers and technology developments. Opt. Eng. 91, 3286.

Romani, R., Miller, A.J., Cabrera, B., Figueroa-Feliciano, E., 1999. First astronomical application of a cryogenic transition-edge-sensor spectrophotometer. ApJ 521, L153.

Russell, C.T. et al., 2007. Exploring the asteroid belt with ion propulsion: dawn mission history, status and plans. Adv. Space Res. 40, 193.

Wen, Y. et al., 2006. Individual photon counting using e2v L3 CCDs for low background astronomical spectroscopy. Proc. SPIE 6276, 44. 